\title{
Compressed State Kalman Filter for Large Systems
}

\begin{abstract}
The Kalman filter (KF) is a recursive filter that allows the assimilation of data in real time and has found numerous applications. In earth sciences, the method is applied to systems with very large state vectors obtained from the discretization of functions such as pressure, velocity, solute concentration, and voltage. With state dimension running in the millions, the implementation of the standard or textbook version of KF is very expensive and low-rank approximations have been devised such as EnKF and SEEK. Although widely applied, the error behavior of these methods is not adequately understood. This article focuses on very large linear systems and presents a complete computational method that scales roughly linearly with the dimension of the state vector. The method is suited for problems for which the effective rank of the state covariance matrix is much smaller than its dimension. This method is closest to SEEK but uses a fixed basis that should be selected in accordance with the characteristics of the problem, mainly the transition matrix and the system noise covariance. The method is matrix free, i.e., does not require computation of Jacobian matrices and uses the forward model as a black box. Computational results demonstrate the ability of the method to solve very large, say $10^{6}$, state vectors.

Key words:

Bayesian Analysis, Geostatistics, Kalman filter, Probability, Statistics
\end{abstract}

\section{Introduction}

The objective of recursive filtering is to update the state of a system that changes in time using the most up-to-date information, such as data from sensors. Applications appear in various engineering, geophysical, and biomedical application. Kalman filter $(\mathrm{KF})$ is a recursive algorithm to 
assimilate data for dynamic systems with state-space structures with whitenoise (independent in time) inputs. The reader is assumed to be familiar with Kalman filter at least at an introductory level. When this recursive filter was originally derived [1] and applied, the focus was on small-state systems that do not pose major computational challenges. However, in geophysical and biomedical applications one increasingly encounters systems with very large state vectors [for example $2,3,4,5]$. These state vectors usually result from the discretization of functions or "images". The dimension of the state vector, $m$ is very large, often $10^{6}$ and up, and the data assimilation problem cannot be handled by the textbook version of Kalman filtering that has computational cost that increases with the square of the state dimension. In terms of computational requirements, the textbook version is $O\left(\mathrm{~m}^{2}\right)$, where the big $\mathrm{O}$ notation indicates the order of magnitude.

This work focuses on problems with the following principal characteristics:

1. The number of state variables (i.e., the quantities that vary in time and describe the state) may be huge whereas the number of observations at every time step is modest.

2. The system is such that the updated covariance matrix of the state can be approximated adequately by reduced rank matrices of manageable size.

The second characteristic, which seems to be assumed in practically all methods dealing with very large systems [as in 6,2], can be attributed to how the dynamic system operates and to how the measurements are collected. In particular, it is the combination of the following factors:

- The system has finite memory in the sense that the effect of past observations gradually decays over time. This is the case when the system has short memory as in parabolic systems with significant diffusion, as in the examples to be presented in this paper.

- The observations have error and the measurement operator is such that small scale fluctuations in the state variables have small effect on observed quantities. Typically the spectrum of the measurement operator, which is the distribution of singular values of the matrix that relates the observations to the unknowns, drops rapidly [as in many tomographic problems, e.g., 7].

As a consequence, only larger-scale features can be resolved [see, for example, 8]. The covariance matrix effectively contains information about a much smaller number of variables than its size would indicate and thus it can be adequately, at least for some classes of applications, approximated by a low-rank matrix. Of course, every approximation involves some loss 
of accuracy vis-à-vis the full algorithm against which it could be compared. However, the accuracy loss may pale in comparison to the statistical error associated with the estimated quantities.

The idea of approximating the state covariance by a reduced-rank matrix is certainly not new. One method that has become popular for the implementation of the Kalman filter to large systems is Ensemble Kalman Filter (EnKF) [9, 10, 11, 12, 13]. This method, which creates an $N$-rank representation through $N$ conditional realizations has major advantages, including that it is straightforward to apply and is highly parallelizable. However, in some applications many realizations are needed to achieve sufficient accuracy. In a different approach, [14] developed a method called the singular evolutive extended Kalman (SEEK) filter. The approach starts with an eigen decomposition that retains a few important modes. An important point is that the starting eigenvectors are then propagated through the state transition equations. The decomposition is renewed to maintain orthogonality. In follow-up work, [15] introduced a variant called singular evolutive interpolated Kalman (SEIK) filter. In [16], it was proposed to keep the basis (i.e., the set of orthogonal vectors) the same rather than modifying them at every period to better reproduce the updated state covariance. They justify the choice on the basis of the application they worked on (ocean models representing a slowly varying system). Additional discussion can be found in [17]. Several other works have examined various aspects of these basic approaches. Perhaps the method closest to this work is that of [4], who also use low-rank approximation of the covariance matrix and also use a fixed basis. The differeneces of this work from [4] is that this work emphasizes algorithmic aspects, i.e., the development of a method that is $O(m)$, and does not focus on a particular basis.

Of course, rank reduction is the only general approach for reducing the computational cost associated with large dimensionality and has been applied in the context of many different problems. For example, [18] has developed a method called Fixed Rank Filtering for the assimilation of spatiotemporal data. However, it must be emphasized that these works are in the context of statistical correlation models whereas the present work presents a complete computational methodology for the efficient utilization of physicsbased models that involve the solution of many coupled ordinary differential equations.

The scope and contributions of this work are as follows. It focuses on linear systems, for two reasons. First, the concept of using compressed or 
reduced rank representations encompasses all large systems, whether linear or nonlinear. It is important to consider this concept on its own and not just as an expedient in dealing with nonlinearity. Second, it is useful to study a method for linear systems before attempting to use it in nonlinear ones, where additional difficulties must be dealt with. This paper presents a complete methodology that is $O(\mathrm{~m})$ in computational and storage requirements, where $m$ is the dimension of the state of the systems that is assumed large. The method uses an expansion similar to the one previously used [14, 16], but the implementation and interpretation differ at some important points. In particular, a salient feature of this method is that it uses a constant basis, as previously suggested by $[16,4]$.

The proposed method, like EnKF and SEEK, can deal with large systems because its computational cost is nearly linear to the size of the state vector, can limit itself only to the use of a simulator that can be implemented as a black box, and is highly parallelizable. However, it will be illustrated that, unlike EnKF, the low rank representation proposed here: $(a)$ is deterministic and is resistant to degradation, (b) uses a relatively accurate, in the spectral norm sense, yet inexpensive way to compute factorization of the posterior (corrected) covariance matrix, $(c)$ takes advantage of fast matrix vector products through hierarchical matrices or FFT [19, 20]. Unlike SEEK, the orthogonal basis is preselected and fixed.

\section{Background}

I will review the basic ideas for completeness and to establish notation.

\section{State-Space Structures}

The evolution in time of many systems encountered in applications can be described by a linear discrete state-space structure, as follows [see textbooks such as 21]:

$$
\begin{gathered}
\boldsymbol{s}(t+\Delta t)=\boldsymbol{\Phi}(t) \boldsymbol{s}(t)+\boldsymbol{w}(t) \\
\boldsymbol{y}(t+\Delta t)=\boldsymbol{H}(t+\Delta t) \boldsymbol{s}(t+\Delta t)+\boldsymbol{v}(t+\Delta t)
\end{gathered}
$$

where the terms are: $s$ is the state of the system; $\boldsymbol{w}$ is stochastic white-noise input disturbance; $\boldsymbol{v}$ is stochastic white-noise observation disturbance; and $\boldsymbol{y}$ is observation. The matrices $\boldsymbol{\Phi}$, and $\boldsymbol{H}$, with appropriate dimensions, are assumed known; more likely, in actual applications, they are not given explicitly but through a method to compute matrix-vector products. I will 
focus on the case that the time step $\Delta t$ is fixed. Known additive inputs like control terms in (1), are omitted as they can be taken care of easily. I will adopt the integer notation where $t=t_{0}+k \Delta t$ is replaced by subscript $k$ to emphasize the discrete-time nature of the problem:

$$
\begin{gathered}
\boldsymbol{s}_{k+1}=\boldsymbol{\Phi}_{k} \boldsymbol{s}_{k}+\boldsymbol{w}_{k} \\
\boldsymbol{y}_{k+1}=\boldsymbol{H}_{k+1} \boldsymbol{s}_{k+1}+\boldsymbol{v}_{k+1}
\end{gathered}
$$

White-noise process indicates no time interdependence or structure. For Gaussian distributions, it suffices to state the following:

$$
\begin{aligned}
E\left[\boldsymbol{w}_{k}\right] & =0 \\
E\left[\boldsymbol{w}_{k} \boldsymbol{w}_{l}^{T}\right] & =\left\{\begin{array}{cc}
\boldsymbol{Q}_{k} & k=l \\
0, & k \neq l
\end{array}\right.
\end{aligned}
$$

$$
\begin{aligned}
E\left[\boldsymbol{v}_{k}\right] & =0 \\
E\left[\boldsymbol{v}_{k} \boldsymbol{v}_{l}^{T}\right] & =\left\{\begin{array}{cc}
\boldsymbol{R}_{k} & k=l \\
0, & k \neq l
\end{array}\right.
\end{aligned}
$$

$$
E\left[\boldsymbol{w}_{k} \boldsymbol{v}_{l}^{T}\right]=\mathbf{0}
$$

There may be interdependence between elements in the state vector or elements in the measurement vector at a certain time, i.e., matrices $\boldsymbol{Q}_{k}$ and $\boldsymbol{R}_{k}$ do not have to be diagonal. For the state-space structure, it is assumed that the white noise processes are independent from the initial condition of the system, $\boldsymbol{s}_{0}$,

$$
\begin{aligned}
E\left[\boldsymbol{s}_{0} \boldsymbol{v}_{k}^{T}\right] & =\mathbf{0} \\
E\left[\boldsymbol{s}_{0} \boldsymbol{w}_{k}^{T}\right] & =\mathbf{0}
\end{aligned}
$$

The above relations, equations (5) through (11), hold for any $k$ and $l$.

The model is versatile and describes many actual problems. The statespace structure is a natural way to represent the evolution of many systems modelled based on conservation laws and constitutive equations and formulated, after special discretization, as systems of ordinary differential equations. The crucial idea is that knowing just $s(k)$ suffices to describe its evolution at $k+1, k+2, \ldots$ 


\section{Kalman Filter Algorithm}

Given that we deal with one period at a time, to simplify notation, the $k$ index will be dropped for system matrices with the understanding that they may be different for each period, keeping the indices only for the state mean and covariance.

\section{Prediction}

Consider that $\boldsymbol{s}_{k}$ given observations up to time step $k$ is Gaussian with mean $\hat{\boldsymbol{s}}_{k \mid k}$ and covariance $\boldsymbol{\Sigma}_{k \mid k}$. The one step prediction problem is to find the probability density function (pdf) of $\boldsymbol{s}_{k+1}$ given the same information and utilizing the state transition equation (3), which is linear. The distribution is Gaussian with mean and covariance:

$$
\begin{gathered}
\hat{\boldsymbol{s}}_{k+1 \mid k}=\boldsymbol{\Phi} \hat{\boldsymbol{s}}_{k \mid k} \\
\boldsymbol{\Sigma}_{k+1 \mid k}=\boldsymbol{\Phi} \boldsymbol{\Sigma}_{k \mid k} \boldsymbol{\Phi}^{T}+\boldsymbol{Q}
\end{gathered}
$$

\section{Updating}

Now, combine two sources of information regarding $\boldsymbol{s}_{k+1}$, one from the prediction and one from the measurement $\boldsymbol{y}_{k+1}$. Using Bayes theorem, with prior from the prediction, the posterior (i.e., conditional on the observations) can be found.

$$
\hat{\boldsymbol{s}}_{k+1 \mid k+1}=\hat{\boldsymbol{s}}_{k+1 \mid k}+\boldsymbol{K}\left(\boldsymbol{y}_{k+1}-\boldsymbol{H} \hat{\boldsymbol{s}}_{k+1 \mid k}\right)
$$

where the linear feedback coefficient $\boldsymbol{K}$ is known as the Kalman gain:

$$
\boldsymbol{K}=\boldsymbol{\Sigma}_{k+1 \mid k} \boldsymbol{H}^{T}\left[\boldsymbol{H} \boldsymbol{\Sigma}_{k+1 \mid k} \boldsymbol{H}^{T}+\boldsymbol{R}\right]^{-1}
$$

and the updated covariance is the predicted covariance minus the reduction through the measurements.

$$
\begin{gathered}
\boldsymbol{\Sigma}_{k+1 \mid k+1}=\boldsymbol{\Sigma}_{k+1 \mid k} \\
-\boldsymbol{\Sigma}_{k+1 \mid k} \boldsymbol{H}^{T}\left[\boldsymbol{H} \boldsymbol{\Sigma}_{k+1 \mid k} \boldsymbol{H}^{T}+\boldsymbol{R}\right]^{-1} \boldsymbol{H} \boldsymbol{\Sigma}_{k+1 \mid k}
\end{gathered}
$$

The cost of implementing the "textbook" version of the Kalman filter is $O\left(m^{2}\right)$, assuming $n<<m$, where $n$ is the number of observations and $m$ is the number of state variables. 


\section{Compressed State Kalman Filter (CSKF) for Linear Systems}

This section presents the proposed algorithm, CSKF, which is an implementation of Kalman filter that scales nearly linearly with $m$. Consider systems with state vectors of size $m$ and measurement vectors of size $n$, where $m$ is potentially huge and $n<<m$. Also, in applications of interest here, the observation matrix $\boldsymbol{H}$ and/or the state transition matrix $\boldsymbol{\Phi}$ have rapidly decaying singular-value spectra. Despite the large size of the state vector, the "information content" is limited so that, for example, the covariance matrix of the $m$-dimensional state can be approximated adequately through much smaller matrices.

The recursions start at $k$ with state with given mean, $\hat{s}_{k \mid k}$, and a given covariance matrix $\boldsymbol{\Sigma}_{k \mid k}$ that is factorized as follows:

$$
\boldsymbol{\Sigma}_{k \mid k}=\boldsymbol{A} \boldsymbol{C}_{k} \boldsymbol{A}^{T}
$$

where $\boldsymbol{A}$ consists of $N$ orthonormal columns, $\boldsymbol{A}^{T} \boldsymbol{A}=\boldsymbol{I}$, while $\boldsymbol{A} \boldsymbol{A}^{T}$ is an orthogonal projector [see, for example, 22], and $\boldsymbol{C}_{k}$ is an $N$-dimensional symmetric positive definite matrix. Consider that $\boldsymbol{A}$ has been preselected and $N$ is typically orders of magnitude smaller than $m$. The ordering is

$$
m>>>n
$$

Equation (17) is an $N$-rank representation of the covariance matrix at time $k$ and I will refer to it as the kernel approximation covariance matrix. The advantages of this approach will become clearer later.

Regarding the very first time step, when the covariance $\boldsymbol{\Sigma}_{k \mid k}$ may be given by a form different from (17), the following procedures can be followed. Given the preselected $\boldsymbol{A}$, one general approach would be to just perform the multiplication

$$
\boldsymbol{C}_{k}=\boldsymbol{A}^{T} \boldsymbol{\Sigma}_{k \mid k} \boldsymbol{A}
$$

However, if the covariance $\boldsymbol{\Sigma}_{k \mid k}$ is a full $m \times m$ matrix, this would be an $O\left(\mathrm{~m}^{2}\right)$ and is not recommended. In practice, the initial state is either already in some low-rank factorized form (such as from an EnKF) or in the form of a stationary-field covariance matrix and the multiplication can be performed with $O(m \log (m))$, which in practice is very similar to $O(m)$, operations using FFT based methods or H-matrices [23, 20, 24]. Thus $\boldsymbol{C}$ can be computed with $O(m)$ operations that are feasible even for large systems. 
The prediction step equations are (12)-(13). Note that the multiplication of $\boldsymbol{\Phi}$ with a vector is usually, in practical applications, done in an efficient way that exploits the structure of the specific dynamic system, as will be discussed in more detail later. The covariance is then

$$
\begin{aligned}
\boldsymbol{\Sigma}_{k+1 \mid k} & =\boldsymbol{\Phi} \boldsymbol{A} \boldsymbol{C} \boldsymbol{A}^{T} \boldsymbol{\Phi}^{T}+\boldsymbol{Q} \\
& =\boldsymbol{A}_{1} \boldsymbol{C} \boldsymbol{A}_{1}^{T}+\boldsymbol{Q}
\end{aligned}
$$

where

$$
\boldsymbol{A}_{1}=\boldsymbol{\Phi} \boldsymbol{A}
$$

which is an $m$ by $N$ matrix. Each column of $\boldsymbol{A}_{1}$ is computed by evaluating the matrix-vector product of $\boldsymbol{\Phi}$ times each column of $\boldsymbol{A}$. Thus, the prediction covariance requires $O(m)$ multiplications and storage. It is worth pointing out that $\boldsymbol{\Sigma}_{k+1 \mid k}$ is not explicitly computed and stored but only the factors $\boldsymbol{A}_{1}$ and $\boldsymbol{C}$ are. Matrix $\boldsymbol{Q}$ usually does not need to be formed but is given by a simple formula [e.g., 24].

Next comes the update step starting with the important cross-covariance term:

$$
\begin{aligned}
& \boldsymbol{\Sigma}_{k+1 \mid k} \boldsymbol{H}^{T} \\
= & \boldsymbol{A}_{1} \boldsymbol{C A}_{2}^{T}+\boldsymbol{Q} \boldsymbol{H}^{T}
\end{aligned}
$$

where

$$
\boldsymbol{A}_{2}=\boldsymbol{H} \boldsymbol{A}_{1}
$$

The computation of $A_{2}$ involves $n \times m \times N$ multiplications. Typically $\boldsymbol{Q}$ has a special structure; it could be the identity matrix or it could correspond to models such as the exponential so that $\boldsymbol{Q}$ is an H-matrix, i.e., can be approximated by data-sparse structures, and the multiplication $\mathbf{Q} \boldsymbol{H}^{T}$ can be performed through $O(m \log m)$ operations using the right software [20, 24]. For large $m$, like $m^{\sim} 10^{6}$, there is little practical difference between $O(m)$ and $O(m \log (m))$ scaling, as one can see by comparing $m$ and $m \log (m)$ in a log-log plot. Thus the cross-covariance term can be computed with almost linear in $m$ cost. Next,

$$
\begin{aligned}
& \boldsymbol{H} \boldsymbol{\Sigma}_{k+1 \mid k} \boldsymbol{H}^{T} \\
= & \boldsymbol{A}_{2} \boldsymbol{C A}_{2}^{T}+\boldsymbol{H} \boldsymbol{Q} \boldsymbol{H}^{T}
\end{aligned}
$$

which is computed at cost of $O(m)$. 
${ }^{237}$ Next, to obtain the Kalman gain $\boldsymbol{K}$, solve a system:

$$
\mathcal{A} \boldsymbol{K}^{T}=\mathcal{B}
$$

which means solving the system

$$
\mathcal{A X}=\mathcal{B} \boldsymbol{A}
$$

246

and for the covariance:

$$
\begin{aligned}
& \boldsymbol{\Sigma}_{k+1 \mid k+1}=\boldsymbol{A}_{1} \boldsymbol{C} \boldsymbol{A}_{1}^{T}+\boldsymbol{Q}- \\
& -\boldsymbol{A} \mathcal{X}^{T}\left(\boldsymbol{A}_{2} \boldsymbol{C} \boldsymbol{A}_{1}^{T}+\boldsymbol{H} \boldsymbol{Q}\right)
\end{aligned}
$$

244

Thus, the update for the mean is

$$
\begin{aligned}
& \hat{\boldsymbol{s}}_{k+1 \mid k+1}=\hat{\boldsymbol{s}}_{k+1 \mid k} \\
& +\boldsymbol{A} \mathcal{X}^{T}\left(\boldsymbol{y}_{k+1}-\boldsymbol{H} \hat{\boldsymbol{s}}_{k+1 \mid k}\right)
\end{aligned}
$$

247 
Finally, in preparation for the next iteration, $\boldsymbol{\Sigma}_{k+1 \mid k+1}$ will be put in the kernel factorized form, equation (17), as described in the next section.

It must be pointed out that in the majority of applications the transition matrix is not explicitly computed by the forward solver. Instead, the timedependent solver computes $\boldsymbol{s}_{k+1}$ from an $\boldsymbol{s}_{k}$,

$$
\boldsymbol{s}_{k+1}=f\left(\boldsymbol{s}_{k}, \boldsymbol{u}\right)
$$

where $\boldsymbol{u}$ may be other inputs, using methods suited to the problem at hand and achieving nearly linear scaling. Thus, the forward-model simulator actually computes the matrix-vector product $\boldsymbol{\Phi} \boldsymbol{x}$, given a vector $\boldsymbol{x}$. The simplest, and usually adequate, approach is numerical differentiation

$$
\boldsymbol{\Phi} \boldsymbol{x}=\frac{\|\boldsymbol{x}\|}{\delta\left\|\boldsymbol{s}_{k}\right\|}\left[f\left(\boldsymbol{s}_{k}+\delta \frac{\left\|\boldsymbol{s}_{k}\right\|}{\|\boldsymbol{x}\|} \boldsymbol{x}, \boldsymbol{u}\right)-f\left(\boldsymbol{s}_{k}, \boldsymbol{u}\right)\right]+O(\delta)
$$

where $\delta$ is a dimensionless number close to the square root of the floatingpoint relative precision $\left(10^{-4}\right.$ for single precision, $10^{-8}$ for double precision).

\section{Covariance Factorization}

There are many methods to produce low-rank factorizations of matrices and in particular of symmetric and definite matrices, like the covariance $\Sigma_{k \mid k}$. The first requirement here is efficiency in computation with reasonable accuracy. Such methods are available, including fast randomized algorithms [25]. However, there is another requirement that is related to the recursive nature of the algorithm. Namely, it is not enough to compute accurately one expansion of $\Sigma_{k \mid k}$ in the form of (17). As the results propagate to the next factorization, of $\Sigma_{k+1 \mid k+1}$, and on to the next, the method used should not be affected by deterioration due to propagation and accumulation of errors.

In doing the factorization, there are a number of strategies:

1. Given $\boldsymbol{\Sigma}_{k+1 \mid k+1}$, compute a low-rank approximation in the general form

$$
\boldsymbol{\Sigma}_{k+1 \mid k+1} \approx \boldsymbol{A}_{k+1} \boldsymbol{C}_{k+1} \boldsymbol{A}_{k+1}^{T}
$$

where $\boldsymbol{A}_{k+1}$ has orthonormal columns. Such an approach appears appealing because it can achieve maximum approximation accuracy but my experience so far has been that it fails the second test. Over time, as information from more observations is collected and reflected in the covariance features, the computation of some columns of the time-varying $\boldsymbol{A}$ may become affected 
by error and degrade to random-looking functions. I will not discuss this approach further in this work.

2. Given $\Sigma_{k+1 \mid k+1}$, compute a low-rank approximation in the general form

$$
\boldsymbol{\Sigma}_{k+1 \mid k+1} \approx \boldsymbol{A} \boldsymbol{C}_{k+1} \boldsymbol{A}^{T}
$$

where $\boldsymbol{A}$ is not recalculated at every step to reproduce $\boldsymbol{\Sigma}_{k+1 \mid k+1}$ but is preselected. Then, the weight of approximating $\boldsymbol{\Sigma}_{k+1 \mid k+1}$ falls on $\boldsymbol{C}_{k+1}$. This approach can be implemented as follows. Take a "reasonable" covariance function, such as the exponential, and perform a reduced-rank singular value decomposition. Keep the eigenvectors to use in $\boldsymbol{A}$. The implementation is quite straightforward and computations are of $O(\mathrm{~m})$ :

$$
\boldsymbol{C}_{k+1}=\boldsymbol{A}^{T}\left(\boldsymbol{\Sigma}_{k+1 \mid k+1} \boldsymbol{A}\right)
$$

3. This is a variation to strategy 2. $\boldsymbol{A}$ is preselected from ready-made orthogonal expansions that are known for their versatility and ease of handling. One such expansion is the one that corresponds to the discrete cosine transform (or, more precisely, to the DCT-2, see [26]). Or, depending on the application, a good choice might be an expansion that corresponds to the eigenvalues of the forward operator (transition matrix $\Phi$ ) if it is symmetric.

Our computational results favor strategies 2 and 3 . Thus, the method developed here differs from previous works that adjust the basis. In particular, one can now discern a major difference from the method in [14]. In their case the covariance is approximated by an approach in the spirit of equation (36) where the inner matrix is diagonal and both the inner and the outer matrices change with the time period. In this work I use a similar expansion but the inner matrix is not diagonal but only positive definite with a computationally reasonable condition number. Only the inner matrix changes with each period while the outer matrix, a set of orthonormal vectors, is fixed. The approach to update the inner matrix is much simpler than performing orthogonalizations, for example by using singular value decomposition (SVD).

\section{Illustrative Example}

Consider, first, a modest-size problem that allows the comparison of the results with those of a full Kalman filter. Also, the example is chosen to be straightforward for a reader to reproduce using the MATLAB computing 
environment. Consider a two-dimensional domain, $[0,1] \times[0,1]$, satisfying a diffusion type of equation that, after discretization in space, becomes the system of ODEs where $\boldsymbol{D}$ is a block tridiagonal sparse matrix resulting from discretization of Poisson's equation with the 5-point operator.

$$
\frac{\partial \mathbf{s}}{\partial t}=-\mathbf{D} \mathbf{s}+\text { noise }
$$

with $\boldsymbol{D}$ that is given by MATLAB's gallery('poisson',nr) command. Integration over time leads to

$$
\boldsymbol{s}_{k+1}=\boldsymbol{\Phi} \boldsymbol{s}_{k}+w_{k}
$$

where $\boldsymbol{\Phi}$ is the matrix exponential of $-\boldsymbol{D} \Delta t$. Thus, $\boldsymbol{\Phi}$ has the same eigenvectors as $\boldsymbol{D}$ and has as eigenvalues the exponential of the eigenvalues of $-\boldsymbol{D} \Delta t$. There are of course good schemes to compute $\boldsymbol{\Phi}$. However, the complete evaluation and storage of the transition matrix becomes prohibitively expensive when $m$ gets large. Note the important point that $\boldsymbol{\Phi}$ is not needed for the implementation of the CSKF method but is needed for the complete or textbook version of KF. To illustrate the use of forward solver as a black box, I will use MATLAB's ode45 solver that computes $\boldsymbol{s}_{k+1}$ given $\boldsymbol{s}_{k}$,

$$
\boldsymbol{s}_{k+1}=\boldsymbol{f}\left(\boldsymbol{s}_{k}\right)+w_{k}
$$

The computational cost of this forward solver increases with $m^{\beta}$, where $\beta$ is slightly higher than 1 , as shown in figure 1 . This near-linear scaling of the forward solver is important for the computational performance of the algorithm. Then, the matrix vector product $\boldsymbol{\Phi} \boldsymbol{x}$ is computed through equation (35). Each such product involves one more call to the forward solver and thus a cost of approximately $O(\mathrm{~m})$.

Next, numerical results will be presented to illustrate the behavior of the CSKF vis-a-vis the full Kalman filter as the rank of the approximation increases. The three metrics used in the comparison will be described later, equations (43)-(45). Here, $\Delta t=0.001$ and the grid is 40 by 40 resulting in $m=1600$ state variables. Consider that there are 75 point observations nonuniformly distributed, and the observation error covariance is $\sigma^{2} \boldsymbol{I}, \sigma=1$. Notice that this case is not favorable for CSKF since the spectrum of singular values of $\boldsymbol{H}$ is constant. Consider $w \sim N(\mathbf{0}, \boldsymbol{Q})$, and $\boldsymbol{Q}$ is from the Matérn family of covariance functions

$$
Q_{i j}=10^{3}\left(1+5 \sqrt{3} d_{i j}\right) \exp \left(-5 \sqrt{3} d_{i j}\right)
$$


where $d_{i j}=\left\|\boldsymbol{x}_{i}-\boldsymbol{x}_{j}\right\|$ is the distance between the centers of two blocks with coordinates given by vectors $\boldsymbol{x}$. Consider the basis from the transition matrix of the problem. More precisely, I take the $N$ orthonormal columns corresponding to the smallest singular values of the Poisson or second-difference matrix $\boldsymbol{D}$. (One intuitive way to think of this is that it is the inverse of $\boldsymbol{D}$ that has covariance-like properties, that is why it is the smallest singular values that are relevant.) For $N=m$, the numerical results of CSKF and KF, both in terms of the mean vector and the covariance matrix, are practically the same (relative differences in the norm of the order of $10^{-11}$ ).

For $N=300$ the results between the complete KF and the CSKF are quite close. For comparing the conditional mean and covariance of KF and CSKF, the following metrics are computed:

$$
\begin{aligned}
S D 1 & =\frac{\left\|\hat{\boldsymbol{s}}_{C S K F}-\hat{\boldsymbol{s}}_{K F}\right\|}{\left\|\hat{\boldsymbol{s}}_{K F}\right\|} \approx 0.05 \\
S D 2 & =\frac{\left\|\boldsymbol{\Sigma}_{C S K F}-\boldsymbol{\Sigma}_{K F}\right\|_{F}}{\left\|\boldsymbol{\Sigma}_{K F}\right\|_{F}} \approx 0.2, \\
S D 3 & =\frac{\operatorname{Tr}\left(\boldsymbol{\Sigma}_{C S K F}-\boldsymbol{\Sigma}_{K F}\right)}{\operatorname{Tr}\left(\boldsymbol{\Sigma}_{K F}\right)} \approx-0.1
\end{aligned}
$$

$S D 1$ is for comparing the relative difference in mean vectors. $S D 2$ is for comparing the relative difference in covariance matrices; the subscript $F$ indicates that the norm is Frobenius; SD3 is the relative underestimation of the variances, the diagonal terms of the covariance matrices. Undervaluation of the variance is hardly surprising in reduced rank filters and is often reported for the EnKF. The results may be considered quite accurate for most practical application. Comparisons among the true image, the KF estimate, and the CSKF estimate are given in figure 2. The two estimates are practically indistinguishable from each other. The error metrics were also computed for $N$ from 200 to 1500 and the results are plotted on figure 3.

Next, I will use the same example to illustrate the applicability of the proposed CSKF method for large $m$. It is worth noting that the cost of implementing the textbook approach increases very rapidly with the size of the state vector. For example, the computational cost performing the matrix multiplications in equation (13) is $O\left(10^{18}\right)$ while the cost of storing a covariance matrix would be $O\left(10^{12}\right)$ bytes when $m=10^{6}$. It thus becomes prohibitively expensive to apply the textbook algorithm for $m$ larger than 
a few thousand. By contrast, I will demonstrate that the CSKF can be computed for state vectors as large as $10^{6}$.

Here, results will be presented for a case where I will also demonstrate in an indirect fashion that the results are accurate. Starting with the covariance of equation (42), a rank-30 approximation of $\boldsymbol{Q}$ was used, computed using a low-rank SVD. This reduced-rank covariance was also used for generating realizations for testing. Then, taking $N=30$, the full $\mathrm{KF}$ and the CSKF should give very close results that should be quite accurate given that the image is quite smooth and there are enough observation points. In other words, the true state and the CSKF estimate of the state should be quite close to each other. This was indeed the case. Figure (4) shows a comparison for $m=10^{6}$. The true and the CSKF estimate are within $1 \%$.

Results from a realistic problem related to $\mathrm{CO} 2$ storage will be presented elsewhere [27].

\section{Concluding Remarks}

This article has presented a computational method, CSKF, to apply the Kalman filter to large systems. CSKF has the following advantages:

- Has computational and storage costs that are linear in the size of the state vector, $O(m)$.

- Uses only direct calls to forward models and is matrix-free, i.e., there is no need to compute or store Jacobian matrices, like the transition matrix $\Phi$.

- Is easy to program for particular applications, with some complementary software for fast matrix-vector multiplications.

A question of practical interest is what is the fixed number of terms that is needed for a satisfactory reduced representation; for example the number 100 or even 50 is often mentioned for EnKF and 20 for SEEK. In some cases, excellent results with respect to both the mean and the covariance can be obtained with a surprisingly low rank. This is particularly the case when a basis is selected that captures the action of both the transition matrix and the covariance of the system noise. But if there is a lot of high-frequency noise and/or the transition matrix is unfavorable, many more terms may 
be needed to obtain good results, particularly in correctly computing the covariance matrices.

Thus, it is difficult to set hard and fast rules related to number of terms needed for satisfactory performance. One must consider the objectives of data assimilation, the quality of the data, and the variability in the image that represents the state. To say that only 100 terms are needed may only reflect the limited complexity or information content in the image. If many informative observations have been assimilated and if interesting features like conditions on complex boundaries, inclusions, etc., must be captured, then it is reasonable to expect that a higher rank will be needed. Still, dealing with a compressed state with dimension in the hundreds is far easier that a state with dimension several orders of magnitude larger.

Acknowledgement Supported by the Department of Energy under Award Number DE-FE0009260.

\section{References}

[1] R. E. Kalman, R. S. Bucy, New results in linear filtering and prediction theory, J. of Basic Eng., Trans. ASME, Series D 83 (3) (1961) 95-108.

[2] G. Liu, Y. Chen, D. Zhang, Investigation of flow and transport processes at the made site using ensemble Kalman filter, Adv. Water. Resour. 31 (2008) 975-986.

[3] A. Chatterjee, A. M. Michalak, J. L. Anderson, K. L. Mueller, V. Yadav, Toward reliable ensemble Kalman filter estimates of CO2 fluxes, Journal of Geophysical Research: Atmospheres 117 (D22) (2012) D22306. doi:10.1029/2012jd018176.

[4] B. Jafarpour, D. B. McLaughlin, Reservoir characterization with the discrete cosine transform, SPE Journal 14 (01) (2009) 182-201.

[5] W. Kurtz, H. J. H. Franssen, H. Vereecken, Identification of time-variant river bed properties with the ensemble Kalman filter, Water Resour. Res. 48 (2012) W10534. doi:10.1029/2011wr011743.

[6] R. H. Reichle, D. B. McLaughlin, D. Entekhabi, Hydrologic data assimilation with the ensemble Kalman filter, Monthly Weather Review 130 (1) (2002) 103-114. doi:10.1175/15200493(2002) $130<0103$ :hdawte $>2.0 . c 0 ; 2$. 
[7] J. Lee, P. K. Kitanidis, Large-scale hydraulic tomography and joint inversion of head and tracer data using the principal component geostatistical approach (pcga), Water Resources Research 50 (7) (2014) 54105427. doi:10.1002/2014wr015483.

[8] G.-H. C. Ng, D. McLaughlin, D. Entekhabi, A. Ahanin, The role of model dynamics in ensemble Kalman filter performance for chaotic systems, Tellus A 63 (5) (2011) 958-977. doi:10.1111/j.16000870.2011.00539.x.

[9] G. Evensen, Sequential data assimilation with a non-linear quasigeostrophic model using Monte Carlo methods to forecast error statistics, J. Geophys Res 99 ('C5') (1994) 10.143-10.162. doi:10.1029/94JC00572.

[10] G. Burgers, P. Jan van Leeuwen, G. Evensen, Analysis scheme in the ensemble Kalman filter, Monthly Weather Review 126 (6) (1998) 17191724. doi:10.1175/1520-0493.

[11] G. Evensen, The ensemble Kalman filter: Theoretical formulation and practical implementation, Ocean Dynamics 53 (2003) 343-367. doi:10.1007/s10236-003-0036-9.

[12] G. Evensen, Data Assimilation: The Ensemble Kalman Filter, Springer, 2006.

[13] Y. Gu, D. S. Oliver, An iterative ensemble Kalman filter for multiphase fluid flow data assimilation, SPE Journal (2007) 438-446.

[14] D. T. Pham, J. Verron, M. C. Roubaud, Singular evolutive Kalman filter with EOF initialization for data assimilation in oceanography, J. Mar. Syst. 16 (1997) 323-340.

[15] D. T. Pham, J. Verron, L. Gourdeau, Singular evolutive Kalman filters for data assimilation in oceanography, C. R. Acad. Sci. Paris 326 (1998) $255-260$.

[16] P. Brasseur, J. Ballabrera-Poy, J. Verron, Assimilation of altimetric observations in a primitive equation model of the gulf stream using a singular evolutive extended Kalman filter, J. Mar. Sys. 22 (4) (1999) 269-294. 
[17] I. Hoteit, D.-T. Pham, J. Blum, A simplified reduced order Kalman filtering and application to altimetric data assimilation in tropical pacific, Journal of Marine systems 36 (1-2). doi:10.1016/S0924-7963(02)00129$\mathrm{X}$.

[18] N. Cressie, T. Shi, E. Kang, Fixed rank filtering for spatiotemporal data, J. Comput. Graph. Stat. 19 (3) (2010) 724-745. doi:10.1198/jcgs.2010.09051.

[19] W. Fong, E. Darve, The black-box fast multipole method, Journal of Computational Physics 228 (23) (2009) 8712-8725. doi:10.1016/j.jcp.2009.08.031.

[20] A. K. Saibaba, S. Ambikasaran, J. Y. Li, P. K. Kitanidis, E. F. Darve, Application of hierarchical matrices to linear inverse problems in geostatistics, Oil Gas Sci. Technol. 67 (5) (2012) 857-875. doi:10.2516/ogst/2012064.

[21] M. S. Grewal, A. P. Andrews, Kalman Theory and Practice, PrenticeHall, Englewood Cliffs, NJ, 1993.

[22] L. N. Trefethen, D. Bau, Numerical Linear Algebra, SIAM, 1997.

[23] W. Nowak, S. Tenkleve, O. Cirpka, Efficient computation of linearized cross-covariance and auto-covariance matrices of interdependent quantities, Mathematical Geology 35 (1) (2003) 53-66.

[24] S. Ambikasaran, J. Li, P. Kitanidis, E. Darve, Large-scale stochastic linear inversion using hierarchical matrices, Computational Geosciences 17 (6) (2013) 913-927. doi:10.1007/s10596-013-9364-0.

[25] N. Halko, P. G. Martinsson, J. A. Tropp, Finding structure with randomness: Probabilistic algorithms for constructing approximate matrix decompositions, SIAM Review 53 (2) (2011) 217-288. doi:10.1137/090771806.

[26] G. Strang, The discrete cosine transform, SIAM Review 41 (1) (1999) 135-147.

[27] J. Y. Li, A. Kokkinaki, E. F. Darve, P. K. Kitanidis, Joint subsurface characterization and dynamic monitoring of CO2 injection through compressed state Kalman filter, Adv. Water Resour. in preparation. 


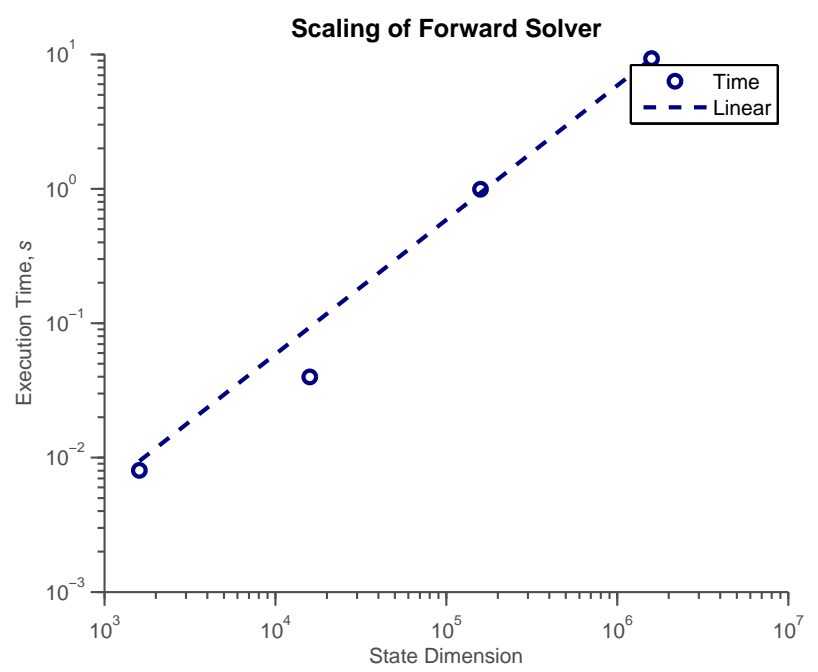

Figure 1: Cost of computing the one-step transition, $\boldsymbol{s}_{k+1}$ from $\boldsymbol{s}_{k}$, for $D t=0.001$. Computed cost is shown through small circles whereas a line of slope 1 is shown for comparison. 
Figure 2: Comparison of true field with KF and CSKF (300) estimates.
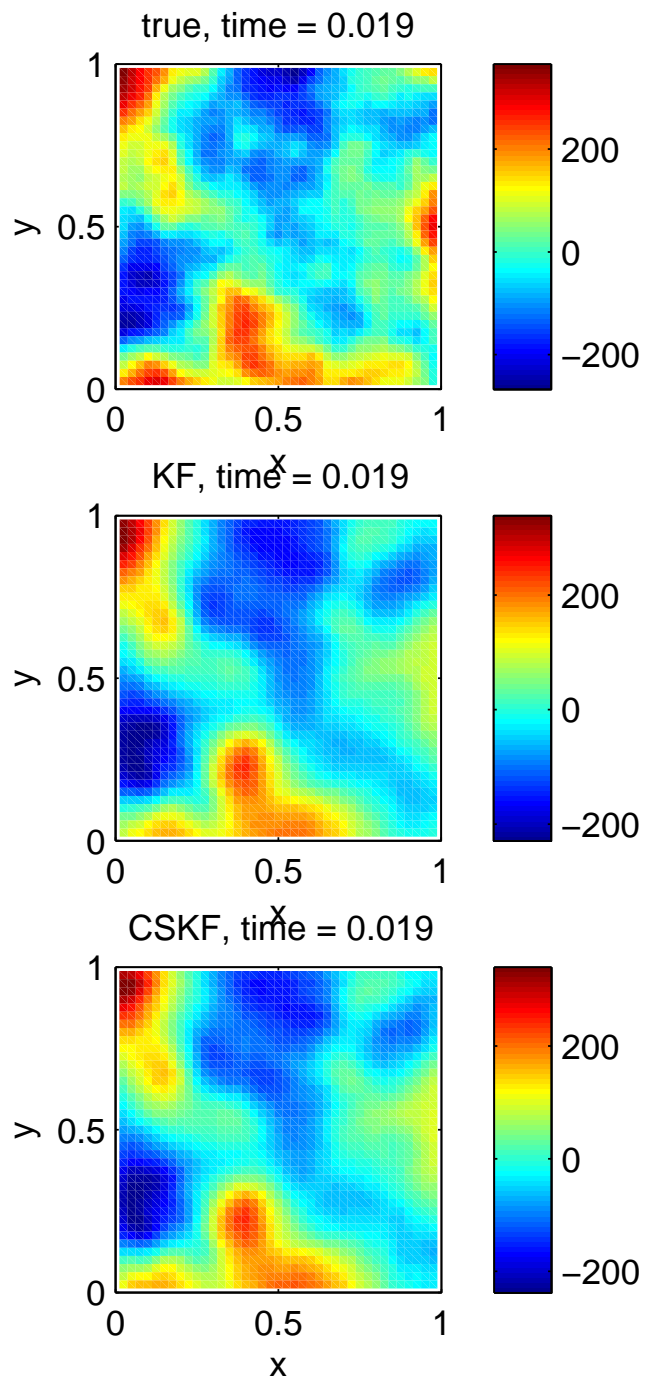


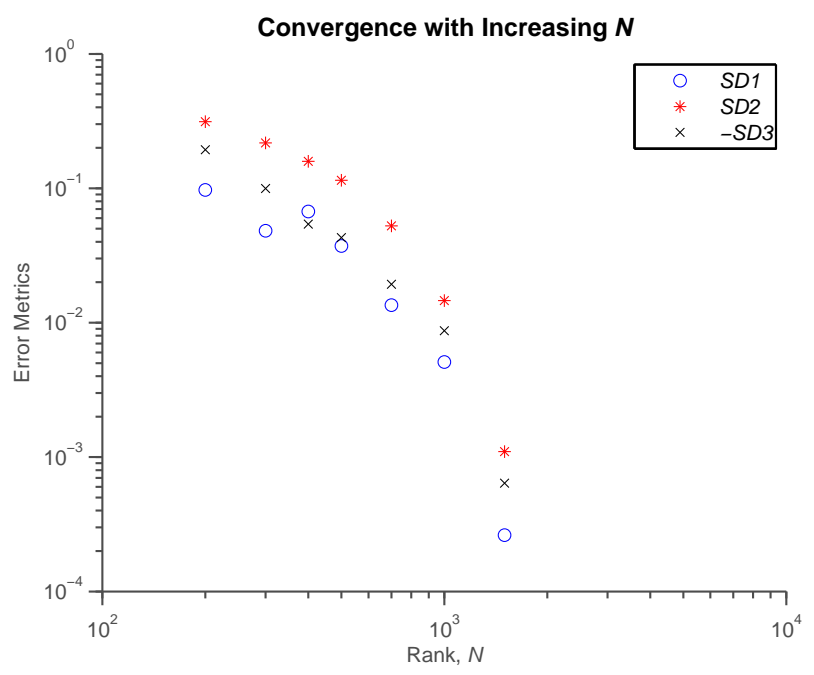

Figure 3: Convergence of error metrics with rank of CSKF, $N$. 

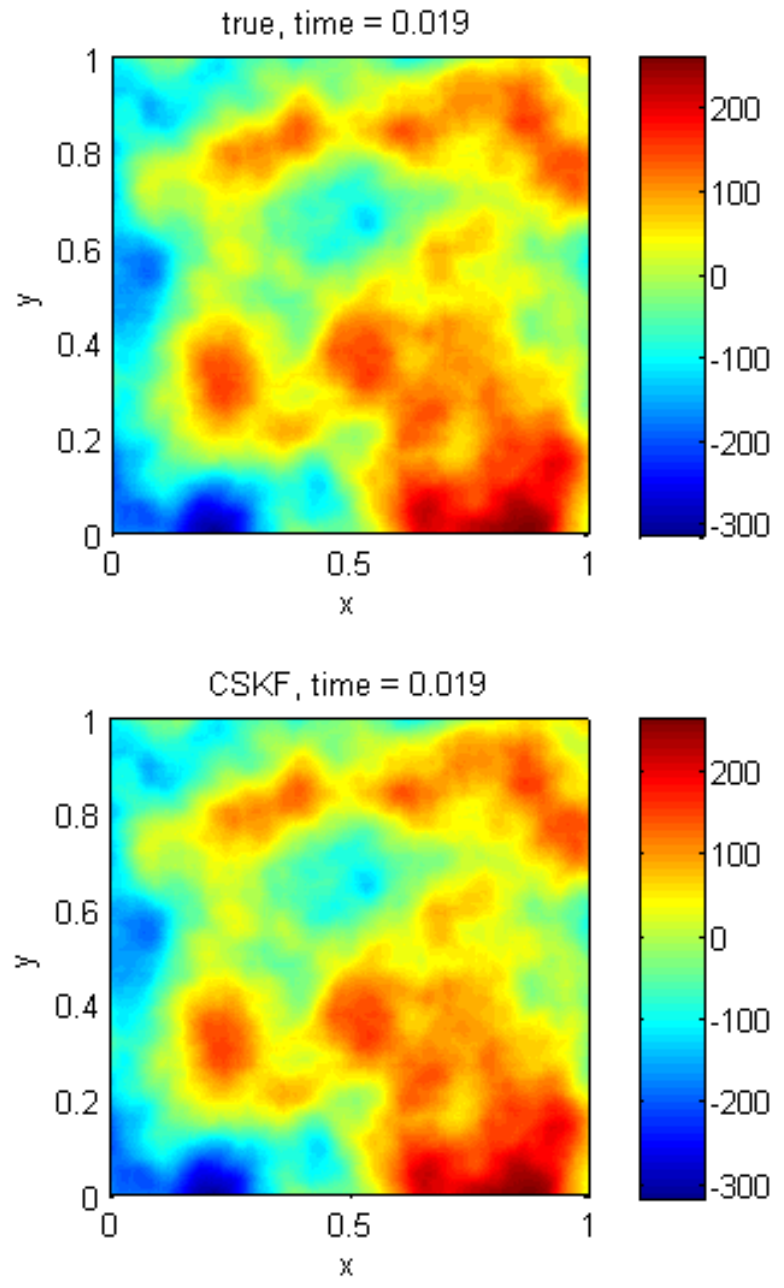

Figure 4: True versus CSKF estimate for $m=10^{6}$. 